\title{
IX. On the diffraction of light by cylinders of large radius
}

\section{Nalinimohan Basu M.Sc.}

To cite this article: Nalinimohan Basu M.Sc. (1918) IX. On the diffraction of light by cylinders of large radius, Philosophical Magazine Series 6, 35:205, 79-97, DOI: 10.1080/14786440108635738

To link to this article: http://dx.doi.org/10.1080/14786440108635738

曲 Published online: 08 Apr 2009.

Submit your article to this journal

Џll Article views: 4

Q View related articles $\square$

4 Citing articles: 4 View citing articles ๘ 
are somewhat analogous to coupled electrical circuits with different inductances or different periods.

2. The case of masses $20: 1$ is seen to be very nearly that of forced vibrations in which the light bob is driven by receiving energy from the heavy bob or driver, while the latter's loss, though equal in energy, entails only a very small decrease of amplitude. The case of masses as $5: 1$ is about midway in character between that of $20: 1$ and equal masses. Eighteen photographic reproductions of double traces are given for unequal masses.

3. It was noticeable on one of the traces that the light bob showed diminntion of amplitude as the trace proceeded. This led to taking resistance into account in the equation of motion. It was also necessary to determine experimentally the actual damping of the light bob when vibrating separately. The theory thus developed and numerically applied fitted the observed facts.

4. In the case of unequal lengths but equal masses, a feebler response and a shorter beat cycle may naturally be expected than if mistuning were absent. Both these effects are quite striking with loose couplings. But with the tighter couplings the effect of mistuning is practically unnoticeable. The theory agrees with this experimental result. Nine sets of double traces are given for the nnequal periods.

5. It is hoped that these methods may be shortly applied to the illustration of important phenomena in other branches of Physics.

Nottingham,

Nov. 19,1917 .

IX. On the Diffraction of Light by Cylinders of Large Radius. By Nalinimohan Basu, M.Sc., Sir Rashbehari Ghosh Research Scholar in the University of Calcutta *.

[Plate III.]

\section{Introduction.}

1. C. F. BRUsh has recently published a paper containing some interesting observations on the diffraction of light by the edge of a cylindrical obstacle $\uparrow$. Brush worked with

* Communicated by Prof. C. V. Raman.

† "Some Diffraction Phenomena: Superposed Fringes," by C. F. Brush. Proceedings of the American Philosophical Society, 1913, pp. 276-282. See also 'Science Abstracts,' No. 1810 (1913). 
cylinders of various radii (the finer ones being screened on one side so as to confine diffraction to the other side only), and observing the fringes formed within a few millimetres of the diffracting edge through a microscope, found that they appeared brighter and sharper with every increase in the radius of the cylinder. The fringes obtained with a smooth rod of one or two centimetres radius differed very markedly from those formed by a sharp edge or by a cylinder of small radius. They were brighter, more numerous, showed greater contrast between the maxima and minima of illumination, and their spacing was different from that given by the usual Fresnel formulæ. Brush also observed that when the radius of the cylinder was a millimetre or more, the fringes did not vanish when the focal plane of the microscope was put forward so as to coincide with the edge of the cylinder. Sharp narrow tringes were observed with the focal plane in this position, becoming broader and more numerous as the radius of the cylinder was increased.

2. To account for these phenomena Brush has suggested an explanation, the nature of which is indicated by the title of his paper. The diffraction-pattern formed by the cylinder is, according to Brush, the result of the superposition of a number of diffraction-patterns which are almost, but not quite, in register. He regards the cylindrical diffracting surface as consisting of a great many parallel elements, each of which acts as a diffracting edge and produces its own fringe-pattern, which is superposed on those of the other elements. Brush has made no attempt to arrive, mathematically or empirically, at any quantitative laws of the phenomena described in his paper. A careful examination of the subject shows that the view put forward by him presents serious difficulties, and is open to objection. One of the defects of the treatment suggested by Brush is that it entirely ignores the part played by the light regularly reflected from the surface of the obstacle at oblique or nearly grazing incidences. I propose in the present paper $(a)$ to describe the observed effects in some detail, drawing attention to some interesting fentures overlooked by Brush; (b) to show that they can be interpreted in a manner entirely different from that suggested by him; and (c) to give a mathematical theory together with the results of a quantitative experimental test.

3. Reference should be made here to the problem of the diffraction of plane electromagnetic waves by a cylinder with its axis parallel to the incident waves. The solution of this problem for a perfectly conducting cylinder has been 
given by J. J. Thomson *, and for a dielectric cylinder by Lord Rayleigh $\dagger$. These solutions are, however, suitable for numerical computation only when the radius of the cylinder is comparable with the wave-length. A treatment of the problem in the case of a cylinder of any radius has been recently given by Debye $\ddagger$. He considers the electromagnetic field round a perfectly reflecting cylinder, whose axis is taken for axis of $z$, with polar co-ordinates $r, \phi$, and waves in the plane $x y$ polarized in the direction of $z$, the electric component in $z$ being $e^{i k x}$. Expressing the disturbance-field in the form

$$
\mathrm{Z}=-\Sigma e^{i n} \frac{\pi}{2} \frac{\mathrm{J}_{n}(\kappa a)}{\mathrm{H}_{n}(\kappa \alpha)} \cdot \mathrm{H}_{n}(\kappa r) \cos n \phi
$$

(in which $J_{n}$ is the usual Bessel function, $H_{n}$ is Hankel's second cylindrical function, and $\kappa=2 \pi / \lambda$ ), Debye transforms the solution into the simple.form

$$
\mathrm{Z}=-\sqrt{\frac{a \cos \frac{\phi}{2}}{2 r}} \cdot e^{-i k\left(r-2 a \cos \frac{\phi}{2}\right)} .
$$

Debye's work is of considerable significance, but his final solution is valid only for points at a great distance from the surface of the cylinder, whereas the phenomena considered in the present paper are those observed in its immediate neighbourhood. No complete mathematical treatment of the subject now dealt with appears to have been given so far.

General Description of the Phenomena.

Fig. 1.
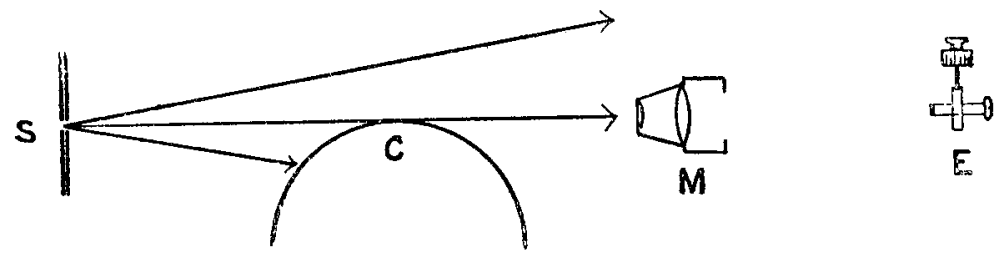

4. The experimental arrangements are those shown in the diagram (fig. 1). Light from a slit $\mathrm{S}$ falls on a polished

* 'Recent Researches in Electricity and Magnetism,' p. 428.

+ Phil. Nag. 1881. 'Scientific Works,' vol. i. p. 534 .

$\mp$ P. Debye, "On the Electromagnetic Field surrounding a Cylinder and the Theory of the Rainbow," Phys. Zeitschr. ix. pp. 775-778, Nov. 1908. Also Deutsch. Phys. Gesell. Verh. 10, 20, pp. 741-749, Oct. 1908; and 'Science Abstracts', No. 258 (1909).

Phil. Mag. S. 6. Vol. 35. No. 205. Jan. $1918 . \quad$ G 
cylinder of metal or glass and passes it tangentially at $\mathrm{C}^{*}$. The axis of the cylinder is parallel to the slit. A collimating lens may, if necessary, be interposed between the slit and the eylinder. The fringes bordering the shadow of the edge $\mathrm{C}$ are observed through the microscope-objective $\mathrm{M}$ and the micrometer eyepiece E. The latter may be placed at any convenient distance from the objective so as to give the necessary magnification. The effects are best seen with monochromatic light obtained by focussing the spectrum of the electric arc on the slit with a small direct-vision prism. For photographic work, the eyepiece $\mathbf{E}$ is removed and replaced by a long light-tight box in front of which the objective $M$ is fixed, and at the other end of which the photographic plate is exposed. Sufficient illumination for photographing the fringes may be secured by using the arc and illuminating the slit by the greenish-yellow light transmitted by a mixture of solutions of copper sulphate and potassium bichromate.

5. The phenomena observed depend on the position of the focal plane of the objective with reference to the diffracting edge of the cylinder, and an interesting sequence of changes is observed as the focal plane of the objective is gradually moved, towards the light, up to and beyond the edge $\mathrm{C}$ (fig. 1) at which the incident light grazes the cylinder. Some idea of these changes will be obtained on a reference to Plate III., figs. I. to VIII., in which the fringes photographed with a cylinder of radius $1.54 \mathrm{~cm}$. are reproduced. (A Zeiss objective of focal length $1.7 \mathrm{~cm}$. was used, and the magnification on the original negative was 135 diameters.)

6. To interpret the phenomena it is convenient to compare them with those obtained by a sharp diffracting edge in the same position. Using the cylinder, it is found that when the focal plane is between the objective and the cylinder, but several centimetres distant from the latter, the fringes are practically of the same type as those due to a sharp diffracting edge. They are diffuse, fow in number (not more than seven or eight being visible even in monochromatic light), and the first bright band is considerably broader and more luminous than the rest. The fringes become narrower (retaining their characteristics) as the focal plane is brought nearer the cylinder till the distance between the two is about two centimetres. At this stage some new features appear; the

* A glass cylinder may be used without incunrenience as the light transmitted through the cylinder is refracted out to one side, and does not enter into the field under observation. Very little light is, in fact, transmitted through the cylinder at oblique incidences. 
contrast between the minima and maxima of illumination becomes greater than in the fringes of the usual Fresnel type, and the number that can be seen and counted in monochromatic light increases considerably. These features become more and more marked as the focal plane approaches the cylinder, and the dark bands then become almost perfectly black. The difference between the intensity of the first maximum and of those following it also becomes less conspicuous. Figs. I., II., and III. in the Plate represent these stages. A considerable brightening-up of the whole field is also noticed as the focal plane approaches the cylinder, but this is not shown in the photographs, as the exposures obtained with the light of the arc were very variable. When the focal plane is within a millimetre or two of the edge at which the incident light grazes the cylinder, a change in the law of spacing of the fringes also becomes evident, the widths of the successive bright bands decreasing less rapidly than in the fringes of the Fresnel type. Fig. IV. in the plate illustrates this feature, which is nost marked when the focal plane coincides with the edge of the cylinder. At this stage, of course, the fringes due to a sharp diffracting edge would vanish altogether.

7. When the focal plane is gradually moved further in, so that it lies between the cylinder and the source of light, some very interesting effects are observed. The fringes contract a little, and the first band, instead of remaining in the fixed position defined by the geometrical edge, moves into the region of the shadow, and is followed by a new system of fringes, eharacterized by intensely dark minima, that appears to emerge from the field occupied by the fringes seen in the previous stages. (See figs. V. and VI.) The first band of this new system is considerably more brilliant than those that follow it. It is evident on careful inspection that the fringes that move into the shadow form an independent system. For it is found that the part of the field from which the new system has separated out appears greatly reduced in intensity in comparison with the part on which it is still superimposed. When the separation of the field into two parts is complete, a few diffraction-fringes of the usual Fresnel type are observed at the geometrical edge of the shadow of the cvlinder. (See figs. VII. and VIII. in the Plate, in which this position is indicated by an arrow.)

8. A comparison of the effects described in the preceding paragraph and of those obtained with a sharp diffracting edge in the same position, furnishes the clue to the correct explanation of the phenomena observed and dealt with in the 
present paper. With a sharp edge, the fringes of the Fresnel type disappear when the focal plane coincides with it, and reappear without alteration of type when the focal plane is between the edge and the source of light. As mentioned above and shown in figs. VII, and VIII. of the Plate, fringes of this type may also be observed with the cylinder when the focal plane is in this position, and in addition we have, inside the shadow, an entirely separate system of fringes characterized by perfectly black minima and a series of maxima with intensities converging to zero. This latter system has nothing in common with the diffraction phenomena of the Fresnel class, and has obviously an entirely different origin. That it is formed exclusively by the light reflected from the surface of the cylinder is proved by the fact that it mav be cut off without affecting the rest of the field by screening the surface. It is accordingly clear that the light reflected from the surface of the cylinder plays a most important part in the explanation of the phenomena, and that the edge of the cylinder grazed by the incident rays alone acts as a diffracting edge in the usual way, and not all the elements of the surface as supposed by Brush. We shall accordingly proceed on this basis to consider the theory of the fringes observed in various positions of the focal plane of the objective.

\section{Theory of the Fringes at the edge of the cylinder.}

9. When the focal plane coincides with the edge at which the incident light grazes the cylinder, it is permissible to regard the fringes seen as formed by simple interference between the light that passes the cylinder unobstructed and the light that suffers reflexion at the surface of the cylinder at various incidences; for, if a sharp diffracting edge be put in the focal plane in the same position, no diffraction-fringes would be visible. The positions of the minima of illumination in the field may be readily calculated.

In fig. 2, let $\mathrm{O}$ be the centre of the cross-section of the cylinder in the plane of incidence, and let $\mathrm{C}$ be the point at which the light grazes the cylinder. It is sufficient for practical purposes to consider the incident beam as a parallel pencil of rays. The ray meeting the cylinder at the point $Q$ is reflected in the direction $Q P$. Let $\angle Q O A=\theta$, so that $\angle \mathrm{OQP}=\frac{\pi}{2}+\theta$, and $\angle \mathrm{OPQ}=\frac{\pi}{2}-2 \theta$. Let $a$ be the radius of the eylinder and $\mathrm{CP}=x$. The difference of path, $\delta$, between the direct ray and the reflected ray reaching the 
point $\mathrm{P}$ is evidently equal to $\mathrm{QP}-\mathrm{RP}$, which can be easily shown to be given by

$$
\delta=a \sin \theta(\sec 2 \theta-1) .
$$

Fig. 2.

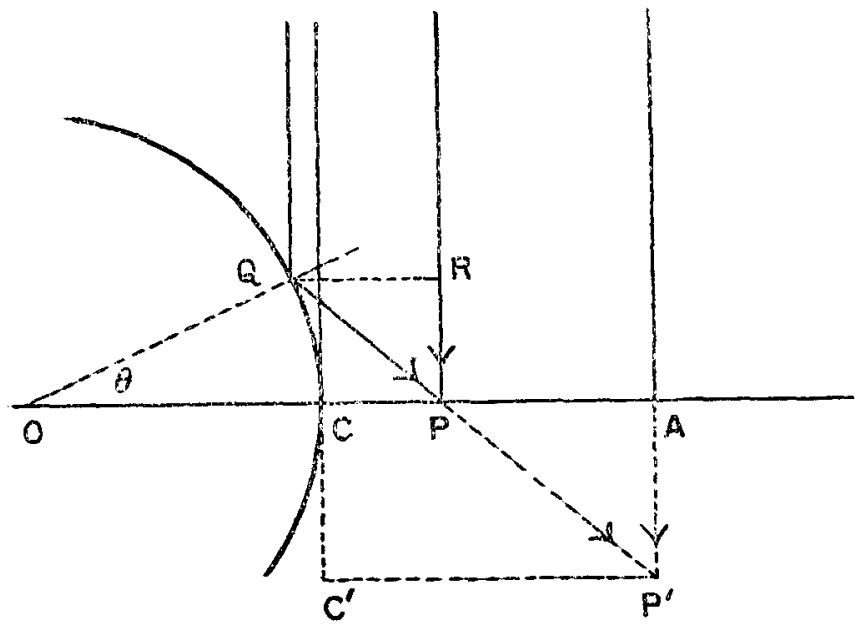

Similarly, we shall have

$$
x=\alpha \sec 2 \theta(\cos \theta-\cos 2 \theta) .
$$

Therefore, neglecting 4 th and higher powers of $\theta$, we have

$$
\delta=2 a \theta^{3}, \text { and } x=3 a \theta^{2} / 2,
$$

so that

$$
\delta=2 a\left(\frac{2 x}{3 a}\right)^{\frac{3}{2}}
$$

Since by reflexion the rays suffer a phase change of half a wave-length, the edge ( will form the centre of a dark band, and the successive minima are therefore given by

$$
x=\left(\frac{n \lambda}{2 a}\right)^{\frac{2}{3}} \cdot \frac{3 a}{2}=\frac{3}{4} \cdot(2 a)^{\frac{1}{3}} \cdot(n \lambda)^{\frac{2}{3}},
$$

where $n=1,2,3$, \&c. The results calculated according to the above theory and those found in experiment are given in Table I. 
TABLE I.

$a=1.54 \mathrm{~cm} . \lambda=6562 \times 10^{-8} \mathrm{~cm}$.

\begin{tabular}{|c|c|c|}
\hline$n$. & $\begin{array}{l}\text { Calculated width } \\
\text { of Band. }\end{array}$ & $\begin{array}{l}\text { Observed width } \\
\text { of Band. }\end{array}$ \\
\hline 1. & $.001775 \mathrm{~cm}$ & $.00174 \mathrm{~cm}$ \\
\hline 2. & .001019 & •00102 , \\
\hline 3. & $\cdot 000875$, & $\cdot 00086$, \\
\hline 4. & .000781 & .00076 \\
\hline 5. & .000717, & 00069 \\
\hline 6. & -000671, & $\cdot 00068$ \\
\hline
\end{tabular}

The discrepancies are within the limits of experimental error. When making these measurements, the focal plane was, in the first instance, set in approximate coincidence with the edge of the cylinder by noting the stage at which a further movement of the focal plane towards the light results in a movement of the fringes into the region of the shadow. There was, however, a slight uncertainty in regard to this adjustment, and the best position of the focal plane was finally ascertained by actual trial.

10. The ratio between the maxima and minima of illumination in the fringes at the edge may readily be calculated. Dividing up the pencil of rays incident on the cylinder into elements of width $a \sin \theta d \theta$ or $a \theta d \theta$ approximately, the width of the corresponding elements of the reflected pencil in the plane of the edge is $d x$, that is, $3 a \theta d \theta$. The amplitude of the disturbance at any point in this plane due to the reflected rays is thus only $1 / \sqrt{ } 3$ of that due to the direct rays, multiplied by the reflecting power of the surface. If the reflecting power be unity (as is practically the case at such oblique incidences), the ratio of the intensities of the maxima and minima is $(1+1 / \sqrt{ } 3)^{2} /(1-1 / \sqrt{ } 3)^{2}$, that is approximately $14: 1$. The dark bands are thus nearly, but not quite, perfectly black.

\section{Theory of the Fringes at the edge of the shadow.}

11. If the fringes be observed in a plane (such as $\mathrm{C}^{\prime} \mathbf{P}^{\prime}$ in fig. 2) which is farther from the source of light than the edge of the cylinder, the diffraction and mutual interference of the direct and the reflected rays have both to be taken into account. Since the, reflected rays form a divergent 
pencil while the incident rays are parallel, the effect of the former at any point sufficiently removed from the cylinder would be negligible in comparison with the effect of the latter. If $d$, the distance of the plane of observation from the edge of the cylinder, be sufficiently large, the problem thus practically reduces to one of simple diffraction of the incident waves by the straight edge C. The positions of the minima of illumination with reference to the geometrical edge of the shadow would then be given approximately by the simple formula

$$
x^{\prime}=\sqrt{2 n d \lambda}=\sqrt{4 n} \sqrt{d \lambda / 2},
$$

where $x^{\prime}=\mathrm{C}^{\prime} \mathrm{P}^{\prime}$ and $d=\mathrm{CC}^{\prime}$,

or with great accuracy by Schuster's formula,

$$
x^{t}=\sqrt{(8 n-1) d \lambda / 4}=\sqrt{(8 n-1) / 2} \sqrt{d \lambda / 2} .
$$

The two formulæ give results which do not differ materialiy except in regard to the first two or three bands, as can be seen from Table II.

\begin{tabular}{|c|c|c|c|c|}
\hline 1. & $\frac{2 .}{\sqrt{4 n}}$ & $\frac{3 .}{\sqrt{(8 n-1) / 2}}$ & $\begin{array}{c}4 . \\
\text { Proportionate } \\
\text { widths of bands } \\
\text { as per column } 2 .\end{array}$ & $\begin{array}{c}5 . \\
\text { Proportionate } \\
\text { widths of bands } \\
\text { as per column 3. }\end{array}$ \\
\hline 1. & $2 \cdot 000$ & 1.871 & $2 \cdot 000$ & $1 \cdot 871$ \\
\hline 2. & $2 \cdot 828$ & $2 \cdot 739$ & 0.828 & 0.868 \\
\hline 3. & $3 \cdot 464$ & $3 \cdot 391$ & 0.636 & 0.652 \\
\hline$t$ & $4 \cdot 000$ & 3.937 & 0.536 & 0.546 \\
\hline 5. & $4: 472$ & $4 \cdot 416$ & 0.472 & $0 \cdot 479$ \\
\hline 6. & $4 \cdot 899$ & $4 \cdot 848$ & $0 \cdot 427$ & $0 \cdot 432$ \\
\hline 7. & $5 \cdot 292$ & $5 \cdot 244$ & 0.393 & $0 \cdot 396$ \\
\hline
\end{tabular}

TABLE II.

12. If $d$ be not large, the intensity of the reflected rays is not negligible. The following considerations enable us to find a simple formula for the positions of the minima of illumination which takes both diffraction and interference into account. We may, to begin with, find the positions of the minima assuming the case to be one of simple interference between the direct and the reflected rays. The expression for the path difference, $\delta^{\prime}$, of the rays arriving at the point $\mathrm{P}^{\prime}$ is readily seen from fig. 2 to be given by the formula

$$
\delta^{\prime}=(d+a \sin \theta)(\sec 2 \theta-1) .
$$


Also,

$$
x^{\prime}=d \tan 2 \theta+a(\cos \theta \sec 2 \theta-1) .
$$

These two relations may, to a close approximation, be written in the form

and

$$
\begin{aligned}
& \delta^{\prime}=2 d \theta^{2}+2 a \theta^{3}, \\
& x^{\prime}=2 d \theta+3 a \theta^{2} / 2 .
\end{aligned}
$$

Putting $d=0$, we get the formula already deduced (see paragraph 9 above) for the fringes at the edge of the cylinder. On the other hand, if $d$ be greater than $a$, we may, to a sufficient approximation, write

$$
\begin{aligned}
& \delta^{\prime}=2 d \theta^{2}, \\
\text { and } \quad x^{\prime} & =2 d \theta,
\end{aligned}
$$

and the positions of the points at which the direct and the reflected rays are in opposite phases are given by the formula

$$
x^{\prime}=\sqrt{2 n \overline{d \lambda}} .
$$

13. But, as remarked above, the simple formula $x^{\prime}=\sqrt{2 n d \lambda}$ also gives the approximate positions of the minima in the diffraction-fringes at a considerable distance from the cylinder, where the effect of the reflected rays is negligible. It is thus seen that the formulæ

and

$$
\left.\begin{array}{rl}
n \lambda & =2 d \theta^{2}+2 a \theta^{3} \\
x^{\prime} & =2 d \theta+3 a \theta^{2} / 2,
\end{array}\right\} \quad \cdot \quad \cdot
$$

suffice to give the approximate positions of the minima of illumination at the edge of the cylinder (at which point the fringes are due to simple interference of the direct and the reflected rays) and also at a considerable distance from it (in which case they are due only to the diffraction of the incident light). A priori, therefore, it would seem probable that the formulæ would hold good also at intermediate points, that is for all values of $d$. That this is the result actually to bo expected may be shown by considering the effect due to the reflected rays at various points in the plane of observation. The reflected wave-front is the involute of the virtual caustic (see fig. 3 below). At the edge $\mathrm{C}$, the radius of curvature of the wave-front is zero, and increases rapidly as we move outwards from the edge of the cylinder. The reflected rays accordingly suffer the most rapid attenuation 
due to divergence in the direction of the incident rays, and less rapid attenuation in other directions. In any plane $\mathrm{C}^{\prime} \mathrm{P}^{\prime}$, therefore, the effect of the reflected light is negligible in the immediate neighbourhood of the point $\mathrm{C}^{\prime}$, and would be most perceptible at points farthest removed from $\mathrm{C}^{\prime *}$, On the other hand, the fluctuations of intensity due to the diffraction of the direct rays are most marked in the neighbourhood of $\mathrm{C}^{\prime}$, that is, for the smallest values of $\theta$. We should accordingly expect to find that when $d$ is not zero, the first few bands are practically identical in position with those due to simple diffraction, and those following are due to simple interference between the direct and the reflected rays. The formulæ given above satisfy both of these requirements. For it is obvious from the manner in which they have been deduced that they satisfy the second requirement. The first requirement is also satisfied, as, by putting $\theta$ small, the formulœ reduce to $n \lambda=2 d \theta^{2}$ and $x^{\prime}=2 d \theta$; or, in other words, $x^{\prime}=\sqrt{2 n d \lambda}$, for the minima of illumination, which is also the usual approximate diffraction formula. Accordingly, the complete formulæ $n \lambda=2 d \theta^{2}+2 a \theta^{3}$ and $x^{\prime}=2 d \theta+3 a \theta^{2} / 2$ would (on eliminating $\theta$ ) give the positions of the minima over the entire field with considerable accuracy.

14. The statements made in the preceding paragraph are, however, subject to an important qualification. 'The validity of the formula obtained rests on the basis that, for large values of $d$, the positions of the minima of illumination are given by the simple relation $x^{\prime}=\sqrt{2 n d \lambda}$. This, however, is only an approximation, as the accurate values are to be found from Schuster's formula (see Table II., above), when the effect of the reflected light is negligible. When $d$ is so large that the formulæ $n \lambda=2 d \theta^{2}+2 a \theta^{3}$ and $x^{\prime}=2 d \theta+3 a \theta^{2} / 2$ give nearly the same positions for the minima as the simple relation $x^{\prime}=\sqrt{2 n d \lambda}$, they should therefore cease to be strictly valid. The actual positions of the minima for such values of $d$ should agree more closely with those given by Schuster's formula, and should, when $\bar{d}$ is very large, agree absolutely with the same. This qualification is, however, of importance only with reference to the first two or three bands obtained for fairly large values of $d$. The differences in respect of the other bands would be negligibly small.

15. To test the foregoing results, measurements were made of the widths of the bright bands for a series of values of $d$

* Debye's formula (loc. cit.) shows that the intensity of the reflected light becomes very small as $\phi$ approaches $\pi$. 
up to $2 \mathrm{~cm}$. Table III. shows the observed values, the values calculated from my formulæ, and the values according to Schuster's formula (which would be valid for a sharp diffracting edge in the same position). To calculate the positions of the minima given by the relations $n \lambda=2 d \theta^{2}+2 a \theta^{3}$, and $x^{\prime}=2 d \theta+3 a \theta^{2} / 2$, the first equation was solved for $\theta$ by Horner's method, and the resulting values substituted in the second equation. The measurements of the width of the first band were rather rough on account of the indefiniteness of its outer edge. The agreement between the observed widths and the widths calculated from my formulæ is seen to be fairly satisfactory for values of $d$ up to $3 \mathrm{~mm}$. For larger values of $d$ the observed widths agree more closely with those calculated from Schuster's formula, as explained in paragraphs 11 and 14 above.

Theory of the Fringes between the edge and the source of light.

16. As already remarked in paragraph 7 , the direct and the reflected pencils tend to separate into distinct parts of the field when the focal plane of the observing microscope is

Fig. 3.

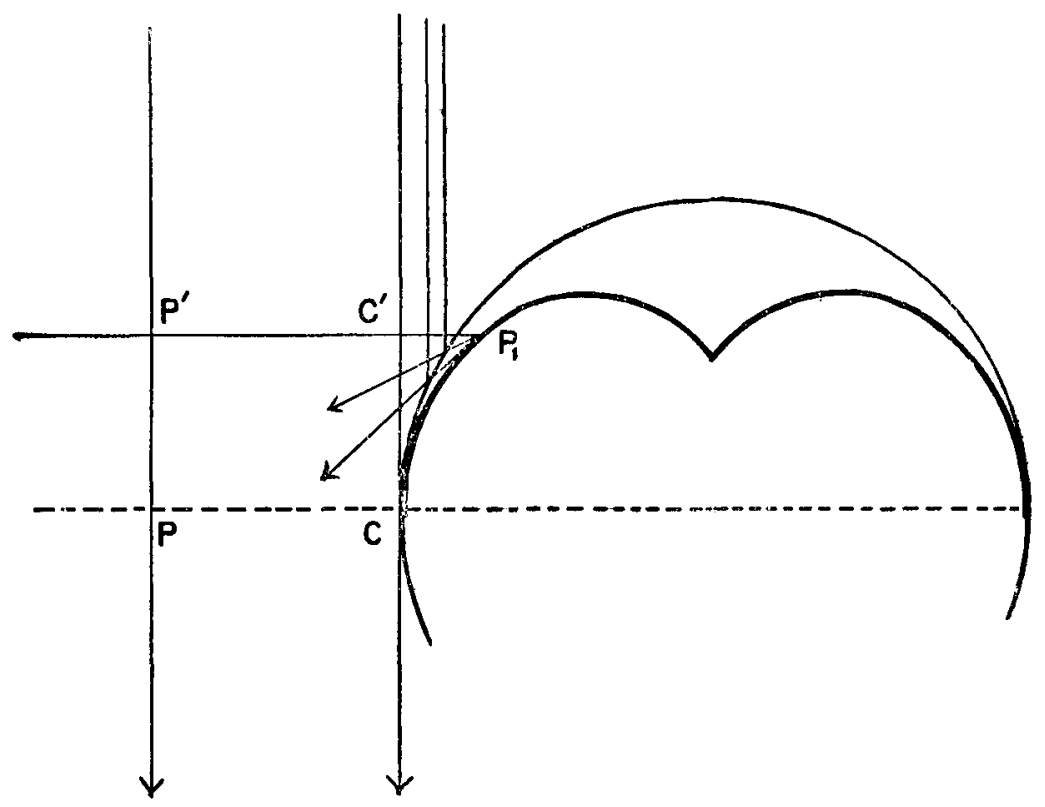

put forward so as to lie between the edge of the cylinder and the source of light. Why this is so will be readily understood on a reference to fig. 3. The rays reflected 


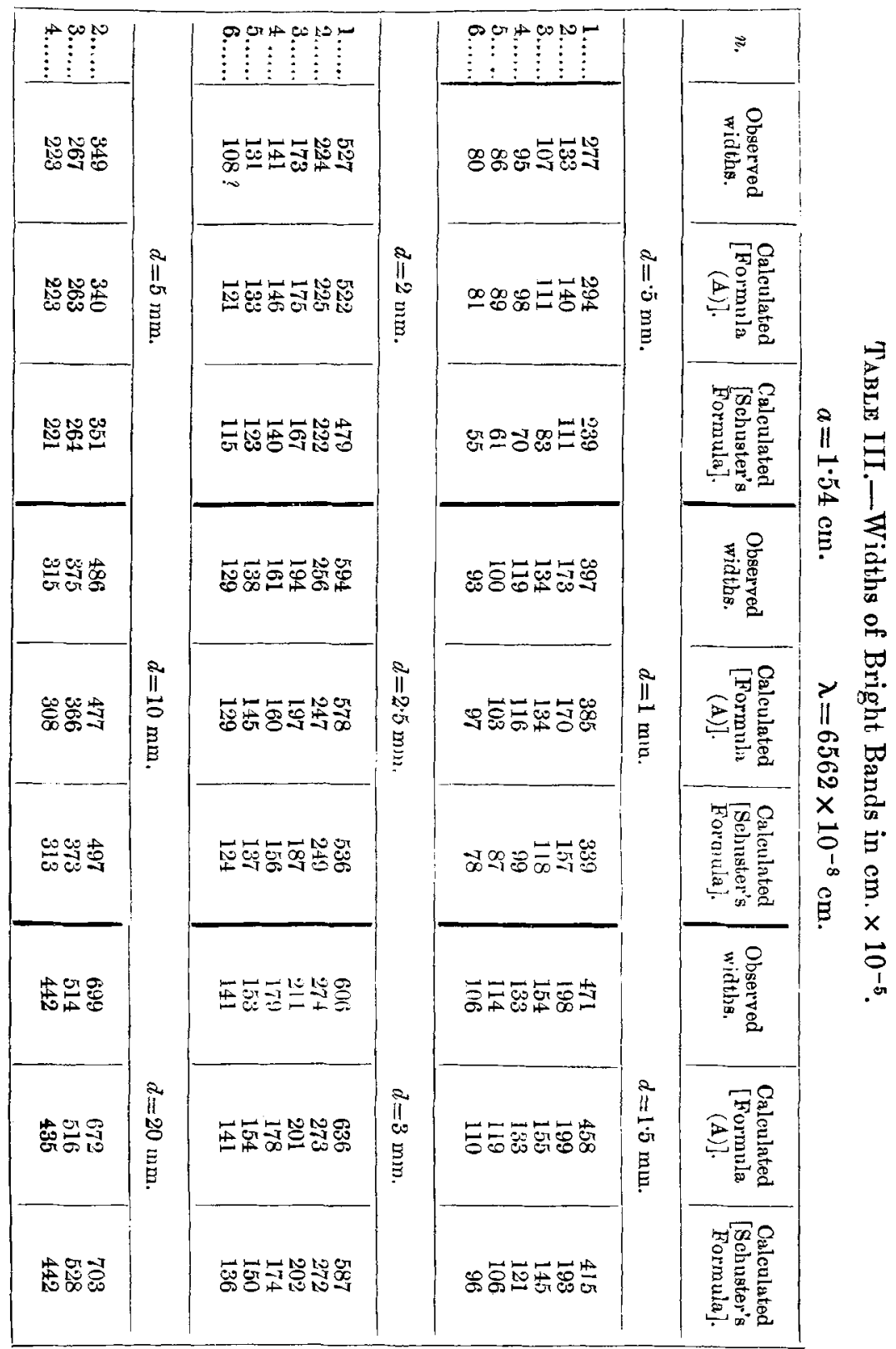


from the surface when produced backwards would touch the enveloping surface which lies within the cylinder. This surface, which is virtually the caustic of the reflected rays, terminates at the edge $\mathrm{C}$ of the cylinder, and when the focal plane of the observing microscope is moved forward from CP to a position $\mathrm{P}^{\prime} \mathrm{C}^{\prime} \mathrm{P}_{1}$ in front of the edge, the boundary of the field on the right-hand side would shift into the region of the shadow, and would, in fact, lie on the surface of the caustic at the point $\mathrm{P}_{1}$. If the focal plane $\mathrm{P}^{\prime} \mathrm{C}^{\prime} \mathrm{P}_{1}$ is considerably forward of $\mathrm{PC}$, the field is seen divided into two parts. The first part $\mathrm{P}^{\prime} \mathrm{C}^{\prime}$ consists of the direct rays alone (the reflected rays meeting $\mathrm{P}^{\prime} \mathrm{C}^{\prime}$ being too oblique to enter into the field of the microscope), and should obviously be bounded at $\mathrm{C}^{\prime}$ by a few diffraction-fringes of the ordinary Fresnel type. The second part of the field $\mathrm{P}_{1} \mathrm{C}^{\prime}$ is due to the reflected rays alone, and requires separate consideration.

17. In the case considered above, that is, when the focal plane is considerably in advance of the edge, the fringesystem within the shadow due to the reflected light is of the same type as that found by Airy in his well-known investigation on the intensity of light in the neighbourhood of a caustic. For the elementary pencils into which the reflected rays may be divided up diverge from points lying along the caustic, and if the point $P_{1}$ at which the focal plane intersects the caustic is sufficiently removed from the edge $\mathrm{C}$ at which the latter terminates, Airy's investigation becomes fully applicable, but not otherwise. The rays emerging from the point $P_{1}$ after passage through the objective of the microscope become a parallel pencil, while pencils emerging from points on either side of $P_{1}$ become convergent and divergent respectively. 'The reflected wave-front after passage through the objective has thus a point of inflexion on either side of which it may be taken to extend indefinitely, provided the are $\mathrm{CP}_{1}$ be long enough. Assuming the focal length to be $f$ and the equation of the wave-front to be $\xi=\mathrm{A} \eta^{3}$, the value of A may be readily found. 'The equation of the caustic is

$$
\left(4 x^{2}+4 y^{2}-a^{2}\right)^{3}-27 a^{4} x^{2}=0 .
$$

From this, or directly by an approximate treatment, it may readily be shown that the radius of curvature of the caustic at the point $\mathrm{C}$ is $\frac{3}{4}$ the radius of the cylinder. For our present purpose, it is thus sufficient to treat the caustic as equivalent to a cylinder of radius $3 a / 4$ touching the reflecting 
surface at C. We have

$$
\mathrm{A}=\frac{1}{6} \frac{d^{3} \xi}{d \eta^{3}}=\frac{1}{6} \frac{d}{d \eta}\left(\frac{d^{2} \xi}{d \eta^{2}}\right)
$$

where $\left(\frac{d^{2} \xi}{d \eta^{2}}\right)$ is the measure of the convergence or divergence of the normals to the wave-front in the neighbourhood of the point of inflexion. Substituting the values obtained from the formulæ of geometrical optics, it is found that

$$
\mathrm{A}=\frac{1}{6} \cdot \frac{3 a}{4} \cdot \frac{1}{f^{3}}=\frac{a}{8 f^{3}} .
$$

The equation of the wave-front is accordingly

$$
\xi=a \eta^{3} / 8 f^{3} \text {. }
$$

The illumination in the fringe-system alongside the caustic is then given by Airy's formula

$$
I=4\left[\int_{0}^{\infty} \cos \frac{\pi}{2}\left(w^{3}+m w\right) d w\right]^{2}
$$

where

$$
m=4 \cdot 2^{\frac{1}{3}} \cdot a^{-\frac{1}{3}} \cdot \lambda^{-\frac{2}{3}} \cdot x_{1},
$$

$x_{1}$ being the distance of any point in the focal plane measured from the point of intersection with the caustic. The integral gives a series of maxima of which the first is the largest, and the rest gradually converge to zero. The minima of illumination are zeroes*. As the focal flane is moved further and further towards the source of light, the fringe-system moves inwards along the caustic, but remains otherwise unaltered.

18. The foregoing treatment of the reflected fringe-system in terms of Airy's theory ceases to be valid when the focal plane is not sufficiently in advance of the edge, and the arc $\mathrm{CP}_{1}$ of the caustic is therefore not large enough. For the reflected wave-front on one side of the point of inflexion then becomes limited in extent, and its equation cannot with sufficient accuracy be assumed to be of the simple form $\xi=\mathrm{A} \eta^{3}$, extending to infinity in either direction. In fact, when the focal plane is at the edge of the cylinder and $\mathrm{CP}_{1}$ is zero, the point of inflexion coincides with the extreme edge of the reflected wave-front. At this stage, of course, the

* Graphs of Airy's integral and references to the literature will be found in an interesting paper by Aichi and Tanakadate (Journal of the College of Science, Tokyo, vol. xxi. Art. 3). 
fringes seen in the field are due only to the interference of the direct and reflected wave-trains. The phenomena noticed as the focal plane is advanced towards the source of light, represent a gradual transition from this stage to one in which Airy's theory becomes fully applicable. In the transition-stages the field of illumination is a continuous whole, of which, however, the different parts present distinct characteristics. First, within the geometrical edge of the shadow, we have a finite number of fringes (one, two, or more according to the position of the plane of observation, but not an indefinitely large number as contemplated by Airy's theory); these may be regarded as the interferencefringes in the neighbourhood of the caustic due to the reflected light alone. Following these we have a long train of fringes due to the interference of the direct and the reflected pencils. The first few of these should ovidently be modified by the diffraction which the direct rays suffer at the edge $\mathrm{C}$ before they reach the observing microscope. Finally, we may also have a part of the field in which the illumination is due only to the direct pencil, the reflected rays not entering the objective of the microscope owing to their obliquity. This part of the field should appear less brightly illuminated than the rest.

19. A complete theoretical treatment of the transitionstages described in the preceding paragraph is somewhat difficult, and has to be deferred to some future occasion. There is no difficulty, however, in calculating the positions of the fringes due to the interference of the direct and the reflected pencils when the focal plane is in advance of the edge, provided the diffraction-effect due to the edge is neglected. It is easily shown that the path-difference between the direct and reflected rays at a point $x^{\prime}$ is given by

$$
\left.\begin{array}{l}
\delta^{\prime}=2 a \theta^{3}-2 d \theta^{2}, \\
x^{\prime}=3 a \theta^{2} / 2-2 d \theta,
\end{array}\right\}, \quad . \quad . \quad .
$$

where $x^{\prime}$ is measured from $\mathrm{C}^{\prime}$ and $d=\mathrm{CC}^{\prime}$. By putting $\delta^{\prime}=n \lambda$ and eliminating $\theta$, the positions of the minima of illumination may be calculated. A complete agreement of the results thus obtained with those found in experiment cannot, however, be expected, as the fringes are narrow and the modifications due to diffraction are not negligible. As regards the fringes alongside the caustic due to the reflected rays, we cannot expect to find a complete agreement between their widths and those found from Airy's theory, so long as the latter is not fully applicable. The divergence, if any, 
should be most marked when the region of the caustic under observation is nearest the edge of the cylinder, and for the fringes which are farthest from the caustic.

20. The for going conclusions have been tested by a series of measurements made with the focal plane in various positions in advance of the edge. To prove that the boundary of the field within the shadow is the caustic and not the surface of the cylinder, measurements were made of the length $C^{\prime} \mathrm{P}_{1}$, the rays incident on the cylinder being a parallel pencil.

$$
\begin{array}{ll}
\text { Observed value of } \mathrm{C}^{\prime} \mathrm{P}_{1} . & \text { Calculated value. } \\
d=1 \mathrm{~mm} \ldots \ldots \cdot 00454 \mathrm{~cm} . & \cdot 00433 \mathrm{~cm} . \\
d=1.3 \mathrm{~mm} . \ldots \cdot 00750 \mathrm{~cm} . & \cdot 00733 \mathrm{~cm} .
\end{array}
$$

The following shows the widths of the fringes observed in the neighbourhood of the caustic when the focal plane was $1.6 \mathrm{~mm}$. in advance of the edge, and the widths calculated from Airy's theory.

Width of fringes in $\mathrm{cm} . \times 10^{-5}$.

$\begin{array}{llllll}\text { Observed .. 159, } & 69, & 56, & 51, & 45, & 43 \\ \text { Calculated... 155, } & 70, & 57, & 50, & 46, & 43 \\ \text { (Airy's theory.) } & & & & & \end{array}$

The agreement in both cases is satisfactory.

21. Table IV. shows the results of measurements made of the fringes in the transition-stages when the focal plane was only a little in advance of the edge, and Airy's theory is not fully applicable. The observed results are in general agreement with the indications of theory set out in paragraph 19. It will be seen that the fringes farthest within the region of the shadow show a fair agreement with Airy's theory, and the others are more nearly in agreement with the widths calculated from formula $(B)$.

\section{Summary and Conclusion.}

22. C. F. Brush has recently published some observations of considerable interest on the diffraction of light by cylindrical edges. The views put forward by him to explain the phenomena, however, present serious difficulties and are open to objection. My attention was drawn to this subject by Prof. C. V. Raman, at whose suggestion the present work was undertaken by $m \theta$ in order to find the true explanation of the effects, and to develop a mathematical theory which would stand a quantitative test in experiment. 'This has now 
Table IV.

Widths of Bright Bands in cm. $\times 10^{-6}$.

$$
a=1.54 \mathrm{~cm} . \quad \lambda=6562 \times 10^{-8} \mathrm{~cm} \text {. }
$$

\begin{tabular}{|c|c|c|c|c|c|}
\hline $\begin{array}{l}\text { Observed } \\
\text { widths. }\end{array}$ & $\begin{array}{c}\text { Calculated } \\
\text { [Airy's } \\
\text { Formula]. }\end{array}$ & $\begin{array}{l}\text { Calculated } \\
\text { [Formula } \\
(\mathrm{B})]\end{array}$ & $\begin{array}{c}\text { Observed } \\
\text { widths. }\end{array}$ & $\left\{\begin{array}{c}\text { Calculated } \\
\text { [Airy's } \\
\text { Formula]. }\end{array}\right.$ & $\begin{array}{l}\text { Calculated } \\
\text { [Formula } \\
\text { (B)]. }\end{array}$ \\
\hline \multicolumn{3}{|c|}{$d=\cdot 2 \mathrm{~mm}$} & \multicolumn{3}{|c|}{$d=\cdot 4 \mathrm{~mm}}$. \\
\hline $\begin{array}{r}1546 \\
852 \\
722 \\
672 \\
622 \\
590\end{array}$ & $\begin{array}{r}1550 \\
696 \\
570 \\
504 \\
461 \\
429\end{array}$ & $\begin{array}{l}894 \\
777 \\
705 \\
637 \\
602\end{array}$ & $\begin{array}{r}1555 \\
777 \\
628 \\
590 \\
566 \\
500\end{array}$ & $\begin{array}{r}1550 \\
696 \\
570 \\
504 \\
461 \\
429\end{array}$ & $\begin{array}{l}754 \\
674 \\
636 \\
594 \\
543\end{array}$ \\
\hline \multicolumn{3}{|c|}{$d=\cdot 6 \mathrm{~mm}}$. & \multicolumn{3}{|c|}{$d=\cdot 8 \mathrm{~mm}$} \\
\hline $\begin{array}{r}1513 \\
700 \\
626 \\
536 \\
493 \\
446\end{array}$ & $\begin{array}{r}1550 \\
696 \\
570 \\
504 \\
461 \\
429\end{array}$ & $\begin{array}{l}\ldots \ldots \\
\ldots \ldots \\
586 \\
549 \\
520 \\
497\end{array}$ & $\begin{array}{r}1466 \\
706 \\
563 \\
503 \\
475 \\
448\end{array}$ & $\begin{array}{r}1550 \\
696 \\
570 \\
504 \\
461 \\
429\end{array}$ & $\begin{array}{l}\ldots \ldots . . \\
\ldots \ldots . \\
\ldots \ldots 08 \\
483 \\
463\end{array}$ \\
\hline
\end{tabular}

been done, and in the course of the investigation various features of importance overlooked by Brush have come to light. The following are the principal conclusions arrived at: (a) The fringes seen in the plane at which the incident light grazes the cylinder are due to the simple interference of the direct and the reflected rays, the positions of the dark bands being given by the formula $x=\frac{3}{4} \cdot(2 a)^{\frac{1}{3}} \cdot(n \lambda)^{\frac{2}{3}}$; (b) the fringes in a plane further removed from the source of light than the cylinder are due to diffraction at the edge grazed by the incident rays but modified by interference with the light reflected from the surface of the cylinder. The positions of the dark bands in these fringes are (to a close approximation) given by the formulæ ${ }^{*} x=2 d \theta+3 \alpha \theta^{2} / 2$, and $n \lambda=2 d \theta^{2}+2 a \theta^{3}$, from which $\theta$ is to be eliminated; (c) when the focal plane of the observing microscope is on the side of the cylinder towards the light, the direct and reflected rays do not both cover exactly the same part of the field, and by putting the focal plane sufficiently forward towards the light,

* This formula is subject to a small correction which is of importance only when $d$ is large. 
they may be entirely separated. When this is the case, the fringes of the ordinary Fresnel type due to the edge of the cylinder may be observed, and inside the shadow we have also an entirely separate system of fringes due to the reflected rays, the first and principal maximum of which lies alongside the virtual caustic formed by oblique reflexion; the distribution of intensity in this system can be found from the well-known integral due to Airy; (d) but when the focal plane is only a little in advance of the edge, the caustic and the reflecting surface are nearly in contact, and Airy's investigation of the intensity in the neighbourhood of a caustic requires modification. It is then found that only a finite number of bands (one, two, three, or more according to the position of tho plane of observation) is formed within the limits of the shadow, and not an indefnitely large number as contemplated by Airy's theory. The rest of the fringes seen in the field are due to the interference of the direct and reflected rays, but modified by diffraction at the edge of the cylinder.

The Indian Association

for the Cultivation of Science,

Calcutta, 8th May, 1917.

X. On Aerial Waves generated by Impact. Part II. By Sudhansurgmar Baneru, M.Sc., Assistant Professor of Applied Mathematics, University of Calcutta* .

[Plate IV.]

\section{Introduction.}

THE origin and characteristics of the sound produced by the collision of two solid spheres were discussed by me at some length in the first paper under the same title that was published in the Philosophical Magazine for July, 1916. It was shown in that paper that the sound is not due to the vibrations set up in the spheres, which in any ordinary material are both too high in pitch to be audible and too faint in intensity, but to aerial waves set up by the reversal of the motion of the spheres as a whole. The intensity of the sound in different directions for the case in which the two spheres were of the same material and diameter, was investigated by the aid of a new instrument which will be referred to as

* Communicated by Prof. C. V. Raman.

Phil. Mag. S. 6. Vol. 35. No. 205. Jan. 1918. 
Bast.

Phil. Mag. Ser. 6, Vol. 35, Pl. III.

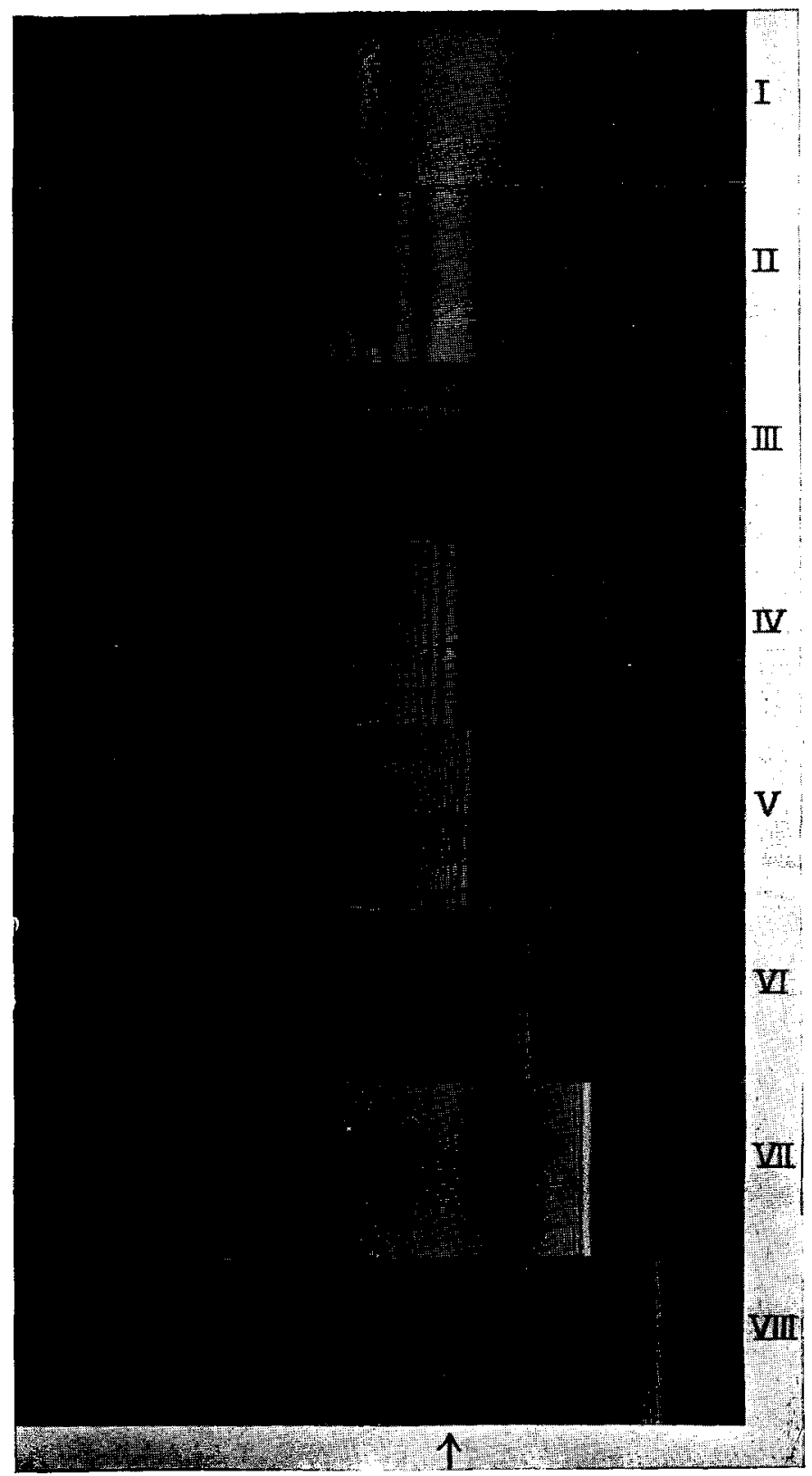

The Diffraction of Light by a Cylinder of radius $1.54 \mathrm{~cm}$. 